Pacific Journal of Mathematics

FINITENESS OF THE RAMIFIED SET FOR BRANCHED 


\title{
FINITENESS OF THE RAMIFIED SET FOR BRANCHED IMMERSIONS OF SURFACES
}

\section{Robert Gulliver}

\begin{abstract}
We shall be concerned with the behavior of a mapping $\pi$ from one oriented compact surface-with-boundary to another, which may fail to be a covering projection in one of two ways. Firstly, $\pi$ need not be a local homeomorphism, although its interior singularities will be of a restricted type, called branch points. Secondly, boundary points may be mapped into the interior, although we shall assume the restriction of $\pi$ to the boundary is injective. We shall show that $\pi$ must then be a local homeomorphism except on a finite set. Moreover, we shall analyze the behavior of $\pi$ near the boundary in sufficient detail to derive a formula relating Euler characteristics of the domain and of the image, with multiplicities, to the total order of branching of $\pi$. These results may be used to study ramification and ramified branch points of parametric minimal surfaces of general topological type.
\end{abstract}

For the present case of a mapping $\pi: M \rightarrow M_{1}$ of one surface, or topological 2-manifold, into another, we may call $\pi$ a branched immersion provided it is locally topologically conjugate to the mapping $g_{m}(z)=z^{m}$ of the unit disk $\Delta$ in the complex plane to itself. That is, for each $p \in M$ there exists an integer $m \geqq 1$, a neighborhood $V$ of $p$ in $M$, a neighborhood $V_{0}$ of $\pi(p)$ in $M_{1}$, and homeomorphisms $h: V \rightarrow \Delta$, $h_{0}: V_{0} \rightarrow \Delta$, with $h(p)=0, h_{0}(\pi(p))=0$ and such that $g_{m} \circ h=h_{0} \circ \pi$. The integer $m-1$ is the order of branching (or order of ramification) of $\pi$ at $p$, denoted $o(p)$. If $o(p)>0$ we call $p$ a branch point; if $o(p)=1, p$ is a simple branch point. For the definition of a branched immersion of a surface into a higher-dimensional manifold, see ([4], Definitions 1.2, 1.6).

The Euler-characteristic formula in the theorem below is a generalization of the Riemann-Hurwitz relation for the case of closed surfaces. The formula has been proved by Ahlfors under the assumption that $\pi$ is a simplicial mapping with respect to appropriate triangulations of the compact surfaces-with-boundary $\bar{M}$ and $\bar{M}_{1}$, with an openness condition at interior edges; this amounts to requiring $\pi$ to be a branched immersion up to the boundary ([1], p. 161, 168). A recent, sheaftheoretic proof has been given by Elwin and Short under the hypothesis that the fibers are constant over components in a finite decomposition of $\bar{M}_{1}([2])$.

One area in which this question is of interest is in the study of ramification of minimal surfaces and of surfaces of prescribed mean 
curvature vector, in conformal parameterization. A mapping $f: M \rightarrow N$ of one manifold into another is said to be ramified if two distinct regular points of $f$ in $M$ define the same germ of submanifold in $N$. A point of $M \cup \partial M$ is called a ramified point of $f$ if the restriction of $f$ to every neighborhood of that point is ramified; a ramified point is necessarily a singular point. If $M$ and $N$ are both two-dimensional and $f$ is a branched immersion, then the notions of branch point and interior ramified point coincide. Now suppose $\bar{M}$ is a compact surface-withboundary, and let $f: \bar{M} \rightarrow N$ be a mapping into a manifold of arbitrary dimension, whose restriction to $M$ is a branched immersion with the unique continuation property (see [4], p. 757), and whose restriction to $\partial M$ is injective. Then the topological space of germs of surface defrned by $f$ at its regular points has a natural compactification $\bar{M}_{1}$; the fundamental theorem of branched immersions states that $\bar{M}_{1}$ is a compact oriented surface-with-boundary, and that the natural quotient mapping $\pi: \bar{M} \rightarrow \bar{M}_{1}$ is a branched immersion in the interior (see Theorem 4.15 of $[3, I])$. Branch points of $\pi$ are precisely the ramified points of $f$. This holds in particular if $f$ is a conformal parameterization of a surface with prescribed mean curvature vector in a riemannian manifold $N$, which maps the boundary injectively into $N$. Thus, the study of the consequences of ramification of $f$ leads naturally to consideration of the mapping $\pi$. The results below will be applied in [3, II] to shed light on ramification of such mappings $f$, and in particular of the minimal surfaces of higher topological type whose existence was proven by Douglas. It should be noted, however, that if the disjoint Jordan curves comprising $f(\partial M)$ are assumed to have a sufficiently high degree of regularity, say class $C^{2}$, then the results of the present paper may be replaced by somewhat simpler arguments exploiting recent results on the regularity of $f$ up to the boundary.

The present work is largely self-contained, relying on a few elementary facts proved in [4]. However, the methods employed will be better understood by a reader familiar with certain concepts and techniques of [4] and of [3, I]. We point out particularly the instructive series of examples in $\$ 5$ of [4].

Branched immersions between surfaces may be characterized by remarkably weak hypotheses, according to a classical theorem of Stoilow ([7], p. 121). Namely, if $\pi: M \rightarrow M_{1}$ is a continuous open mapping between surfaces, and $\pi$ is light, that is, $\pi^{-1}\left(p_{0}\right)$ is totally disconnected for each $p_{0} \in M_{1}$, then $\pi$ is a branched immersion. Thus the result of the present paper implies that a light continuous mapping $\pi: \bar{M} \rightarrow \bar{M}_{1}$ between compact oriented surfaces-with-boundary, whose restriction to $M$ is open and whose restriction to $\partial M$ is injective, is a local homeomorphism except on a finite set in $\bar{M}$, at each point of which there is a well-defined order of branching. 
Notation. When the notation $\bar{M}$ or $\bar{M}_{k}$, etc., is used to denote a surface-with-boundary, we shall write $M$ or $M_{k}$ for the surface consisting of its interior points, $\partial M$ or $\partial M_{k}$ for its boundary. If $\bar{M}$ is a surfacewith-boundary, then an open set $U \subset M$ is itself a surface; however, its closure $\bar{U}$ need not be a surface-with-boundary, and $\partial U=\bar{U} \backslash U$ need not be a 1-submanifold. A connected oriented compact surface-withboundary $\bar{M}$ may be obtained from a sphere by attaching a certain number $g$ of handles, and removing a number of disjoint open disks; $g$ is the genus of $M$. If $M$ is not connected, then its genus is the sum of the genera of its connected components. The Euler characteristic $\chi(\bar{M})=$ $2 c-2 g-k$, where $c$ is the number of components of $M, g$ the genus of $M$, and $k$ the number of boundary components. The restriction of a mapping $\pi: \bar{M} \rightarrow \bar{M}_{1}$ to a subset $U \subset M$ is denoted $\pi \mid U$. In the context of a branched immersion $\pi: \bar{M} \rightarrow \bar{M}_{1}$, we shall use the notation $B_{r}$ for the set of ramified points of $\pi$ in $\bar{M} ; B=B_{r} \cap M$ denotes the set of interior branch points, and $B_{\partial}=B_{r} \cap \partial M$ is the set of ramified boundary points.

For a continuous mapping $\pi: M \rightarrow M_{1}$ of one oriented surface onto another, we may define the Brouwer degree as an integer-valued function $\operatorname{deg}(\pi)$, defined at those points $p_{0} \in M_{1}$ such that there is a compact neighborhood $U$ of $p_{0}$ in $M_{1}$ whose pre-image $\pi^{-1}(U)$ is a compact subset of $M$. That is, $\operatorname{deg}(\pi)$ is defined on the complement of the set of limits of images of properly divergent sequences in $M$. If, as in the case treated in this paper, $M$ is the interior of a compact surface-withboundary $\bar{M}$ and $\pi$ extends continuously over $\bar{M}$, then $\operatorname{deg}(\pi)$ is defined on $M_{1} \backslash \pi(\partial M)$. Now $\operatorname{deg}(\pi)\left(p_{0}\right)$ may be computed as follows: let $\varphi$ be any smooth approximation to $\pi$, with respect to some pair of differentiable structures, which has $p_{0}$ as a regular value. Then $\operatorname{deg}(\pi)\left(p_{0}\right)$ is the number of points in $\varphi^{-1}\left(p_{0}\right)$ at which $\varphi$ preserves orientation, minus the number at which $\varphi$ reverses orientation. We note that if $\pi$ is a branched immersion, then with respect to the appropriate orientations of $M$ and $M_{1}, \varphi$ may be chosen to preserve orientation at all points (see, e.g., Lemma 2 below). Suppose $\pi_{t}: M \rightarrow M_{1}$ defines a homotopy, that is, a jointly continuous one-parameter family of mappings. Then for fixed $p_{0} \in M, \operatorname{deg}\left(\pi_{t}\right)\left(p_{0}\right)$ is constant as a function of $t$ on any interval where it is defined: the proof given in [5], pp. 27-9, for the case that $\partial M$ and $\partial M_{1}$ are empty, may be extended without difficulty to the present case. It follows that for a mapping $\pi: M \rightarrow M_{1}, \operatorname{deg}(\pi)$ is constant on connected components of its (open) domain of definition. In fact, one needs only the following lemma: if $U$ is a connected open subset of a differentiable manifold $M_{1}$ and $p, q \in U$, then there exists a homotopy of diffeomorphisms $h_{t}: M_{1} \rightarrow M_{1}$, such that $h_{t}(U)=U$ for $0 \leqq t \leqq 1, h_{0}$ is the identity, and $h_{1}(p)=q$. The proof of this lemma is completely analogous to the case $U=M_{1}$ given by Milnor ([5], pp. 22-4). 
1. Finiteness of interior branching. Our first lemma illustrates the power of the requirement of injectivity on the boundary. The lemma includes, as special cases, Lemma 6.13 of [4] and Lemma 2.6 of $[3, I]$, and is proved in a fashion similar to the proof of the former. We shall indicate its proof here, in the interest of completeness.

Lemma 1. Let $\Delta$ denote the unit disk in the plane, $\Delta^{+}, \Delta^{-}$and I its intersection with the open upper half-plane, the open lower half-plane and the horizontal axis, respectively. Suppose $\bar{M}$ is a surface-with-boundary (not necessarily compact), and $M_{1}$ is a surface. Let $\pi: \bar{M} \rightarrow M_{1}$ be a continuous mapping, whose restriction to $M$ is a branched immersion, and whose restriction to $\partial M$ is injective. Let $\partial M$ and $I$ be oriented so that $M$ and $\Delta^{+}$, respectively, lie to the left. Then for any $p \in \partial M$ there is an arbitrarily small simply-connected neighborhood $V \cup K$ of $p$ in $\bar{M}$, where $V \subset M, K \subset \partial M$; an integer $m \geqq 1$; a neighborhood $V_{0}^{\circ}$ of $\pi(p)$ in $M_{1}$; and a homeomorphism $g: V_{0} \rightarrow \Delta$; such that $g \circ \pi$ maps an arc of $K$ homeomorphically onto $I$ in orientation-preserving fashion, and such that $\operatorname{deg}(g \circ \pi \mid V)$ is defined on $\Delta \backslash I$, has the value $m$ on $\Delta^{+}$, and has the value $m-1$ on $\Delta^{-}$. Moreover, we may choose $V_{0}$ to be disjoint from $\pi(\partial V \cap$ $M)$, and we may choose $V$ so that $(V \cup K) \cap \pi^{-1}(\pi(p))=\{p\}$.

Proof. Choose a simply-connected neighborhood $U_{0}$ of $p_{0}=\pi(p)$ in $M_{1}$. Let $V \cup K$ be tentatively chosen so that (1) $\partial V=\gamma \cup K$, where $K$ is an arc of $\partial M$ and $\gamma$ is a Jordan arc in $M$ connecting the two end points of $K$; (2) $\pi(\bar{V}) \subset U_{0}$; (3) $p_{0} \notin \pi(\gamma)$. Then $\pi(K)$ is a Jordan arc in $U_{0}$ passing through $p_{0}$ : it follows from the Jordan separation theorem that $p_{0}$ has an arbitrarily small neighborhood $V_{0}$ which is homeomorphic to $\Delta$ under a homeomorphism $g: V_{0} \rightarrow \Delta$ which maps $\pi(K) \cap V_{0}$ to $I$ in orientation-preserving fashion. We choose $V_{0}$ small enough that it is disjoint from $\pi(\bar{\gamma})$. Then $\operatorname{deg}(g \circ \pi \mid V)$ is well-defined on $\Delta \backslash I$, and its constant value $m$ on $\Delta^{+}$is one greater than its value on $\Delta^{-}$, as may be seen from the winding-number characterization of the Brouwer degree. Now since $\pi$ is an open mapping on $V$, the cardinality of $\pi^{-1}\left(q_{0}\right)$ is a lower semi-continuous function of $q_{0} \in M_{1}$ (cf. Lemma 3.26 of [4]). In particular, $V \cap \pi^{-1}\left(p_{0}\right)$ consists of at most $m-1$ points. We now make the final choice of $V \cap K$, choosing $V$ small enough that $V \cap \pi^{-1}\left(p_{0}\right)$ is empty, and choose $V_{0}$ accordingly.

COROLlaRy 1. Suppose $\bar{M}, \bar{M}_{1}$ are compact oriented surfaces-withboundary, $\pi: \bar{M} \rightarrow \bar{M}_{1}$ a mapping which is a branched immersion in $M$ and whose restriction to $\partial M$ is injective. Then $\operatorname{deg}(\pi)$ is bounded, and its maximum and minimum differ by at most the number of components of $\partial M$. 
Proof. First observe that for any curve $\gamma$ in $M_{1}$, the function $\operatorname{deg}(\pi)$ changes along $\gamma$ by exactly the intersection number of $\gamma$ with $\pi(\partial M)$. In fact, the contribution from interior neighborhoods is locally unchanged, while the contribution from a boundary neighborhood changes by 1 as $\pi(\partial M)$ is crossed from right to left, according to Lemma 1. Choose $p_{0} \in M_{1} \mid \pi(\partial M)$. Then since $\bar{M}$ is compact, $\operatorname{deg}(\pi)\left(p_{0}\right)$ is finite: any smooth approximation to $\pi$ is proper, so that only finitely many points are mapped to any regular value. On the other hand, for any point $q_{0} \in M_{1}$ there is a curve $\gamma$ from $p_{0}$ to $q_{0}$ which crosses each component of $\pi(\partial M)$ at most once. Therefore $\operatorname{deg}(\pi)\left(q_{0}\right)$ is at most equal to $\operatorname{deg}(\pi)\left(p_{0}\right)$ plus the number of components of $\partial M$.

In the proof of Proposition 1 below, it will be convenient to work with branched immersions, all of whose branch points are simple. This will be made possible by the following lemma.

Lemma 2. Let $\pi: V \rightarrow V_{0}$ be a branched immersion between surfaces $V$ and $V_{0}$, with exactly one branch point $q \in V$ of order $o(q)=$ $m-1$. Then $\pi$ is homotopic to a branched immersion $\pi_{1}: V \rightarrow V_{0}, \pi_{1}=\pi$ outside of an arbitrarily small neighborhood of $q$, and which has exactly $m-1$ branch points, all of order 1 .

Proof. In an arbitrarily small neighborhood of $q, \pi$ is topologically conjugate to the mapping $g(z)=z^{m}$ of the unit disk onto itself. It suffices, therefore, to prove the statement of the lemma for $\pi=g$, $q=0$. Now choose $m-1$ distinct points $z_{1}, \cdots, z_{m-1}$ on the unit circle in the complex plane. For $0 \leqq t \leqq 1$, we define an analytic function $h_{t}$ by the conditions $h_{t}(0)=0$ and

$$
h_{t}^{\prime}(z)=m\left(z-t z_{1}\right)\left(z-t z_{2}\right) \cdots\left(z-t z_{m-1}\right) .
$$

Now let $\varphi(r)$ be a smooth real-valued function for $0 \leqq r \leqq 1$, with $\varphi(r)=1$ for $r \leqq 1 / 4$ and $\varphi(r)=0$ for $r \geqq 1 / 2$. Define $g_{t}(z)=$ $\varphi(|z|) h_{t}(z)+(1-\varphi(|z|)) g(z)$. Then $g_{0}=h_{0}=g$. Also, for $|z|<1 / 4$, $g_{t}(z)=h_{t}(z)$, so that for $t<1 / 4, g_{t}$ has the $m-1$ simple branch points $t z_{1}, \cdots, t z_{m-1}$. For $|z|>1 / 2, g_{t}(z)=g(z)$ and is an immersion. It may be computed that if $t$ is sufficiently small, then $g_{t}$ is an immersion on the annulus $1 / 4 \leqq|z| \leqq 1 / 2$ also.

Since the Brouwer degree is constant under homotopy, Lemma 2 gives an explicit formula for the degree of a branched immersion $\pi: M \rightarrow M_{1}:$

$$
\operatorname{deg}(\pi)\left(p_{0}\right)=\Sigma\left\{o(p)+1: p \in M, \pi(p)=p_{0}\right\}
$$


With these preliminaries at hand, we are ready to prove the finiteness of interior branching, as a first step toward the finiteness of the set of all ramified points.

Proposition 1. Suppose $\bar{M}, \bar{M}_{1}$ are compact oriented surfaces-withboundary, $\pi: \bar{M} \rightarrow \bar{M}_{1}$ a continuous mapping which is a branched immersion in $M$ and which maps $\partial M$ injectively. Then the set $B \subset M$ of interior branch points of $\pi$ is finite.

Proof. We shall find an upper bound for the total order of branching in an appropriately chosen neighborhood of any point in $\partial M$. Since $B$ is a discrete subset of $M$, the conclusion will then follow from the compactness of $\bar{M}$.

Consider a point $p \in \partial M$. Applying Lemma 1, we may find a simply-connected neighborhood $V \cup K$ of $p$ in $\bar{M}$, and a neighborhood $V_{0}$ of $\pi(p)$ in $M_{1}$ which is separated into two simply-connected components $V_{0}^{+}$and $V_{0}^{-}$by $\pi(K)$, such that $\operatorname{deg}(\pi \mid V)$ has the constant values $m$ on $V_{0}^{+}$and $m-1$ on $V_{0}^{-}, V_{0}$ is disjoint from $\pi(\partial V \cap M)$, and such that $(V \cup K) \cap \pi^{-1}(\pi(p))=\{p\}$. Let $W \cup L$ be a neighborhood of $p$ with $\pi(W \cup L) \subset V_{0}, W \subset V$ and $L \subset K$. We shall show that the total order $O_{W}$ of branching of $\pi$ in $W$ is at most $(m-1)^{2}$.

We first apply Lemma 2 to see that without loss of generality, it may be assumed that $\pi$ has only simple branch points in $W$. Namely, $B$ is discrete; in an arbitrarily small neighborhood of each branch point $q$ of order $o(q)>1$, we replace $\pi$ by a branched immersion homotopic to it, having exactly $o(q)$ simple branch points in this neighborhood, and we leave $\pi$ unchanged outside this neighborhood. Further, we may readily modify $\pi$ so that for each branch point $q \in V, \pi(q) \notin \pi(K)$; and so that for distinct branch points $q, q^{\prime} \in V, \pi(q) \neq \pi\left(q^{\prime}\right)$. Observe that these modifications do not change the total order of branching of $\pi$ in $W$. Let $O_{W}^{ \pm}$be the number of branch points $q \in W$ of $\pi$ (as now modified) with $\pi(q) \in V_{0}^{ \pm}$: thus $O_{w}=O_{w}^{+}+O_{w}^{-}$.

We shall first find an estimate for $O_{W}^{+}$. Write $\nu=O_{W}^{+}$: there are distinct simple branch points $p_{1}, \cdots, p_{\nu}$ in $W$ with distinct images $P_{J}=\pi\left(p_{J}\right) \in V_{0}^{+}, 1 \leqq j \leqq \nu$. Choose a point $Q \in V_{0}^{+} \backslash \pi(B)$, and let the $m$ points of $V \cap \pi^{-1}(Q)$ be denoted $q_{1}, \cdots, q_{m}$. For each $P_{j}, 1 \leqq j \leqq \nu$, choose a closed curve $\gamma_{j}:[0,1] \rightarrow V_{0}^{+} \backslash \pi(B), \gamma_{J}(0)=\gamma_{\jmath}(1)=Q$, such that $\gamma_{J}$ has winding number 1 around $P_{,}$but has winding number 0 around $\pi\left(p^{\prime}\right)$ for every branch point $p^{\prime} \in V$ other than $p_{r}$. We choose the curves $\gamma_{1}, \cdots, \gamma_{\nu}$ to be disjoint except at $Q$. Now since $\pi(\partial V)$ is disjoint from $V_{0}^{+}$, it may be seen that $\pi: V \rightarrow M_{1}$ is locally a covering map over $\gamma_{J}([0,1])$. Thus for each $k, 1 \leqq k \leqq m$, there is a unique lifting $\delta_{j k}:[0,1] \rightarrow V$ with $\pi \circ \delta_{j k}=\gamma_{\jmath}$ and $\delta_{j k}(0)=q_{k}$. Because $p_{\jmath}$ is a simple branch point, it follows that there are two integers $r=r(j), s=s(j)$, with 
$1 \leqq r<s \leqq m$, such that $\delta_{j r}(1)=q_{s}, \delta_{j s}(1)=q_{r}$, and for $r \neq k \neq s, \delta_{j k}(1)=q_{k}$. In fact, we may observe that $P_{j}$ has exactly $m-1$ pre-images in $V$ under $\pi$, namely $p_{j}$ plus $m-2$ distinct regular points, since $\operatorname{deg}(\pi \mid V)\left(P_{j}\right)=m$. But on an appropriate small punctured neighborhood of $p_{\jmath}, \pi$ is a two-to-one covering map onto its image.

Now suppose, for contradiction, that $O_{w}^{+}=\nu>m(m-1) / 2$. There are exactly $m(m-1) / 2$ ways to choose distinct pairs $r, s$ with $1 \leqq r<s \leqq$ $m$. Thus our supposition implies that the same pair is chosen twice. That is, for a certain pair $i, j$ of numbers with $1 \leqq i<j \leqq \nu$, we have $r(i)=r(j)$ and $s(i)=s(j)$. We shall write simply $r=r(i)=r(j)$ and $s=s(i)=s(j)$. Let a closed curve $\delta:[0,2] \rightarrow V$ be defined by $\delta(t)=\delta_{\text {Ir }}(t)$ and $\delta(1+t)=\delta_{\text {Is }}(t)$ for $0 \leqq t \leqq 1$ : this construction will be denoted $\delta=\delta_{i r}+\delta_{\rho s}$. Observe that $\pi \circ \delta=\gamma_{i}+\gamma_{j}$.

For clarity in the following discussion, we shall assume there is a simple arc $\gamma$ passing through $Q$ such that the closed curve $\gamma_{t}+$ $\gamma_{j}:[0,2] \rightarrow V_{0}^{+}$traverses $\gamma$ twice, once simply in each direction, and otherwise is disjoint from $\gamma$. This may be achieved without changing the homotopy classes of $\gamma_{t}$ and $\gamma_{J}$ in $V_{0}^{+} \backslash \pi(B)$ with base point $Q$. Now since $\gamma_{t}$ and $\gamma_{s}$ are disjoint except at $Q$, there is a closed curve $\tilde{\gamma}:[0,2] \rightarrow\left(V_{0}^{+} \mid \pi(B)\right) \cup\{\pi(p)\}, \quad \tilde{\gamma}(0)=\tilde{\gamma}(2)=\pi(p)$, which is disjoint from $\gamma_{l}((0,1))$ and $\gamma_{J}((0,1))$, and which meets $Q$ exactly once at $Q=\tilde{\gamma}(1)$, with $\tilde{\gamma}(t)$ crossing from one side of $\gamma$ to the other at $t=1$. Since $\tilde{\gamma}$ misses $\pi(B), \pi$ is locally a covering projection over $\tilde{\gamma}((0,2))$. Therefore, there is a unique lifting $\tilde{\delta}:(0,2) \rightarrow V$ with $\pi \circ \tilde{\delta}=\tilde{\gamma}$ and $\tilde{\delta}(1)=q_{r}$. Note that $\tilde{\delta}$ leaves every compact subset of $V$ as $t \rightarrow 0$ and as $t \rightarrow 2$, since $\pi\left(p^{\prime}\right) \neq \pi(p)$ for $p^{\prime} \in V$. Meanwhile $V$ is simplyconnected, which implies that $\delta$ has intersection number zero with any closed curve in $V$. But $\tilde{\delta}$ intersects the closed curve $\delta$ exactly once, at $\tilde{\delta}(1)=q_{r}$, a regular point of $\pi$, at which point $\tilde{\delta}$ crosses from one side of $\delta$ to the other: that is, the intersection number of $\delta$ with $\delta$ is \pm 1 . This contradiction shows that $O_{w}^{+} \leqq m(m-1) / 2$.

Similarly, since $\operatorname{deg}(\pi \mid V)$ has the constant value $m-1$ on $V_{0}^{-}$, it may be shown that $O_{W}^{-} \leqq(m-1)(m-2) / 2$. Therefore, the total order of branching of $\pi$ in $W$,

$$
O_{w}=O_{w}^{+}+O_{w}^{-} \leqq(m-1)^{2},
$$

and, in particular, $\pi$ has at most $(m-1)^{2}$ branch points in $W$.

2. Behavior near ramified boundary points. We now turn our attention to the boundary ramified set $B_{\partial}$. Having established the finiteness of the interior branch set $B$, we may restrict attention to a neighborhood of any given boundary point which is disjoint from $B$, that is, on whose interior part $\pi$ is a local homeomorphism. Under the 
hypothesis that the restriction of $\pi$ to the boundary is injective, the behavior of $\pi$ near any boundary point can be described quite precisely. The following proposition will be applied to an appropriate neighborhood $U \cup K$ of a boundary point, where $U \subset M$ and $K$ is an arc of $\partial M$.

Proposition 2. Suppose $U \cup K$ is an oriented surface with boundary $K$, and let $M_{1}$ be an oriented surface. Let $\pi: U \cup K \rightarrow M_{1}$ be a continuous mapping which is a local homeomorphism on $U$ and which maps $K$ injectively. Denote $S^{\prime}=U \cap \pi^{-1}(\pi(K))$. Consider any point $p \in K$. Then there is a neighborhood $V \cup K_{1}, V \subset U$ and $K_{1}$ an arc of $K$, which may be chosen arbitrarily small; an integer $m \geqq 1$; and a Jordan curve $\gamma_{0}$ in $M_{1}$, which bounds a disk $D_{0}$; with the following properties. (1) $P=\pi(p) \in D_{0}$. (2) $\gamma=V \cap \pi^{-1}\left(\gamma_{0}\right)$ consists of a single Jordan arc, with endpoints $a$ and $b$ on $K$; the union of $\gamma$ with the arc of $K$ between $a$ and $b$ bounds a disk $D=V \cap \pi^{-1}\left(D_{0}\right)$. (3) $S^{\prime} \cap D$ is the disjoint union of a family of $2 m-2$ disjoint Jordan arcs $\sigma_{1}, \cdots, \sigma_{m-1}, \tau_{1}, \cdots, \tau_{m-1}$, each tending to $p$ at one end and to distinct points of $\gamma$ at the other. (4) $\gamma_{0}$ meets $\pi(K)$ in exactly two points, $A=\pi(a)$ and $B=\pi(b)$. (5) Finally, $\pi(K)$ separates $D_{0}$ into two disks, $D_{0}^{+}$and $D_{0}^{-}$, so that $\operatorname{deg}(\pi \mid D)$ has the constant values $m$ on $D_{0}^{+}$and $m-1$ on $D_{0}^{-}$.

Proof. We first refer to Lemma 1 , with $\bar{M}=U \cup K$, to see that there exists a simply-connected, relatively compact neighborhood $V \cup$ $K_{1}$ of $p$ in $U \cup K, \quad V \subset U$ and $K_{1} \subset K$, an integer $m \geqq 1$, and a simply-connected neighborhood $V_{0}$ of $P$ in $M_{1}, V_{0}$ disjoint from $\pi(\partial V \cap$ $U)$, so that $V_{0}$ is divided into components $V_{0}^{+}$and $V_{0}^{-}$by the Jordan arc $\pi(K)$ and $\operatorname{deg}(\pi \mid V)$ has the constant values $m$ on $V_{0}^{+}$and $m-1$ on $V_{0}^{-}$. Moreover, we may assume $\left(V \cup K_{1}\right) \cap \pi^{-1}(\pi(p))=\{p\}$. Let $\gamma_{0}$ be any closed Jordan curve in $V_{0}$ which has $P$ in its interior $D_{0}$, and which meets $\pi(K)$ in exactly two points, $A$ and $B$, at each of which $\gamma_{0}$ crosses between $V_{0}^{+}$and $V_{0}^{-}$. Since $\pi$ is a local homeomorphism on $V$, we see that $\gamma=V \cap \pi^{-1}\left(\gamma_{0}\right)$ is the disjoint union of Jordan curves and arcs. Observe that the only limit points of $\gamma$ in $\bar{V}$ are the unique points $a$ and $b$ on $K$ with $\pi(a)=A, \pi(b)=B$, since $\pi(U \cap \partial V)$ is disjoint from $V_{0}$. Since $\bar{V}$ is compact, it follows that $\gamma \cup\{a, b\}$ is compact.

Let $\gamma_{1}$ be any connected component of $\gamma$, and choose $q \in \gamma_{1}$ with $\pi(q) \notin \pi(K)$. Beginning from the point $\pi(q)$ on $\gamma_{0}$, we may construct curves $\delta_{0}, \quad \tilde{\delta}:[0,1] \rightarrow M_{1} \quad$ with $\quad \delta_{0}(0)=\tilde{\delta}_{0}(0)=\pi(q), \quad \delta_{0}(1)=P \quad$ and $\tilde{\delta}_{0}(1) \notin \pi(\bar{V})$, so that $\delta_{0}((0,1))$ and $\tilde{\delta}_{0}((0,1])$ are disjoint from $\gamma_{0} \cup$ $\pi(K)$. Let $\delta:\left[0, t_{0}\right) \rightarrow V$ and $\delta:\left[0, \tilde{t}_{0}\right] \rightarrow V$ be the unique maximal liftings of $\delta_{0}$ and $\tilde{\delta}_{0}$, that is, with $\delta(0)=\tilde{\delta}(0)=q, \pi \circ \delta=\delta_{0}$ and $\pi \circ \tilde{\delta}=\tilde{\delta}_{0}$. Now $\delta_{0}((0,1])$ lies in the interior of $\gamma_{0}$ and hence in $V_{0}$. Thus $\delta(t)$ remains in a compact subset of $V \cup K_{1}$ as $t \rightarrow t_{0}$; by a standard argument, 
one may show that $t_{0}=1$. Further, since $\left(V \cup K_{1}\right) \cap \pi^{-1}(P)=\{p\}, \delta$ has a continuous extension to $[0,1]$ given by $\delta(1)=p$. On the other hand, as $t \rightarrow \tilde{t}_{0}, \tilde{\delta}(t)$ tends to $\partial V \cap U$, and $\tilde{t}_{0}<1$. This shows that $p$ may be reached from one side of $\gamma_{1}$, and $\partial V \cap U$ from the other, by means of paths which do not cross $\gamma$.

Now if any component $\gamma_{1}$ of $\gamma$ is closed, then it separates $V$ into an interior and exterior by the Jordan curve theorem, and both $p$ and $\partial V \cap U$ would be in the exterior. This contradiction shows that $\gamma$ has no closed components in $V$. However, $\gamma$ is a one-dimensional submanifold of $V$, and $\gamma \cup\{a, b\}$ is compact. Thus every component of $\gamma$ is an arc from $a$ to $b$. Each such arc must separate $\bar{V}$ into two components, one containing $p$ and the other containing $\partial V \cap U$. Finally, if there were two components $\gamma_{1}$ and $\gamma_{2}$ of $\gamma$, then since $\gamma_{1}$ and $\gamma_{2}$ are disjoint, $\gamma_{2}$ must lie in one component or the other of $V \backslash \gamma_{1}$. If $\gamma_{2}$ lies in the component containing $p$, then every path from $\gamma_{1}$ to $p$ crosses $\gamma_{2}$, contradicting the result of the above paragraph. Otherwise, every path from $\gamma_{2}$ to $p$ crosses $\gamma_{1}$, which is again a contradiction. This shows that $\gamma$ consists of a single Jordan arc from $a$ to $b$. Let $D$ be the open set in $V$ bounded by $\gamma$ and the arc of $K$ between $a$ and $b$.

Now consider a point $q$ moving from $a$ to $b$ along $\gamma$ : since $\pi$ is a local homeomorphism in $V, \pi(q)$ must move along $\gamma_{0}$ in a strictly monotone fashion. For any $Q \in \gamma_{0} \cap V_{0}^{+}$, there are precisely $m$ points in $V \cap \pi^{-1}(Q)$, and these must all lie on $\gamma$, so that $Q$ is crossed exactly $m$ times. Similarly, a point $Q^{\prime} \in \gamma_{0} \cap V_{0}^{-}$is crossed exactly $m-1$ times. It follows that $A$ and $B$ are crossed exactly $m$ times, counting $a$ and $b$. Thus we may write $\pi^{-1}(A) \cap V=\left\{a_{1}, \cdots, a_{m-1}\right\}$ and $\pi^{-1}(B) \cap V=$ $\left\{b_{1}, \cdots, b_{m-1}\right\}$, where these points occur along $\gamma$ in alternating order: $a, b_{1}, a_{1}, b_{2}, \cdots, a_{m-1}, b$.

Let $\sigma, \tau:[0,1] \rightarrow V_{0}$ be homeomorphisms into the Jordan arc $\pi(K)$, with $\sigma(0)=A, \tau(0)=B, \sigma(1)=\tau(1)=P$. Then $\sigma$ and $\tau$ may be lifted uniquely to give maximal curves $\sigma_{k}:\left[0, s_{k}\right) \rightarrow V, \tau_{k}:\left[0, t_{k}\right) \rightarrow V$, with $\sigma_{k}(0)=a_{k}, \tau_{k}(0)=b_{k}, \pi \circ \sigma_{k}=\sigma$ and $\pi \circ \tau_{k}=\tau, 1 \leqq k \leqq m-1$. Note that these $2 m-2$ arcs are disjoint Jordan arcs, since $\pi$ is a local homeomorphism on $V$. Denote $\sigma_{0}, \tau_{0}:[0,1] \rightarrow K$ the unique liftings, $\pi \circ \sigma_{0}=\sigma, \pi \circ \tau_{0}=\tau$. We shall show $s_{k}=t_{k}=1,1 \leqq k \leqq m-1$. First observe that $\sigma_{k}(t)$ leaves every compact subset of $V$ as $t \rightarrow s_{k}$. However, since $\sigma((0,1]) \subset D_{0}, \sigma_{k}\left(\left(0, s_{k}\right)\right) \subset D$, so the only possible cluster point of $\sigma_{k}(t)$ would be on the arc of $K$ between $a$ and $b$. Any such cluster point is mapped by $\pi$ to $\sigma\left(s_{k}\right)$, so by the injectivity of $\pi$ on $K$, the only possible cluster point is $\sigma_{0}\left(s_{k}\right)$. If $s_{k}<1$, then $\sigma_{k}\left(\left[0, s_{k}\right]\right)$ separates $D$ into two components, such that the arc of $\gamma$ between $a$ and $a_{k}$ cannot be connected to $\tau_{0}([0,1])$ by a path in $D$ unless that path crosses $\sigma_{k}\left(\left[0, s_{k}\right]\right)$. Meanwhile, $\tau_{1}$ connects $b_{1}$ to $\tau_{0}\left(t_{1}\right)=\tau_{1}\left(t_{1}\right)$; but $b_{1}$ lies on the arc of $\gamma$ between $a$ and $a_{k}$, so that $\tau_{1}$ must cross $\sigma_{k}$, say at $\tau_{1}(t)=\sigma_{k}(s)$. But then 
$\tau(t)=\pi \circ \tau_{1}(t)=\pi \circ \sigma_{k}(s)=\sigma(s)$, which can only happen if $t=s=1$. Therefore $s_{k}=1$ for $1 \leqq k \leqq m-1$, and similarly $t_{k}=1$.

We shall show next that $D=V \cap \pi^{-1}\left(D_{0}\right)$. Observe that $\pi(D)$ is connected and disjoint from $\gamma_{0}$, and that $P \in \pi(D)$; therefore, $\pi(D) \subset$ $D_{0}$, and hence $D \subset V \cap \pi^{-1}\left(D_{0}\right)$. Conversely, suppose $q \in V$ and $\pi(q) \in$ $D_{0}$. Then $\pi(q)$ is one endpoint of an arc $\eta_{0}:(0,1) \rightarrow D_{0} \backslash \pi(K), \eta_{0}(0)=$ $\pi(q), \eta_{0}(1)=P . \quad \eta_{0}$ has a unique lifting $\eta:[0,1) \rightarrow V$ with $\eta(0)=q$, as may be seen via a standard argument. But $\eta(t) \rightarrow p$ as $t \rightarrow 1$, since $(V \cup K) \cap \pi^{-1}(P)=\{p\}$. Meanwhile $\eta_{0}$ is disjoint from $\gamma_{0}$, so that $\eta$ cannot cross $\gamma$. Therefore $q \in D$.

It remains to show that $S^{\prime} \cap D$ is the union of the $2 m-2$ disjoint Jordan arcs $\sigma_{k}((0,1))$ and $\tau_{k}((0,1)), 1 \leqq k \leqq m-1$. First observe that since the function $\operatorname{deg}(\pi \mid V)$ is lower semi-continuous, a point $Q \in$ $\pi(K)$ can have at most $m-1$ pre-images in $V$. Now consider $q \in S^{\prime} \cap$ $D$. Since $q \in V$, we have $\pi(q) \neq P$. Thus we may write either $\pi(q) \neq$ $\sigma(t)$ or $\pi(q)=\tau(t)$ for some $t, 0<t<1$. In either case, the $m-1$ distinct points $\sigma_{1}(t), \cdots, \sigma_{m-1}(t)$ or $\tau_{1}(t), \cdots, \tau_{m-1}(t)$ are all in $V \cap \pi^{-1}(\pi(q))$, which, according to the degree argument, contains at most $m-1$ points. Therefore $q$ is one of these.

Definition. For $\pi: U \cup K \rightarrow M_{1}$ as in Proposition 2, and for any $p \in K$, observe that the integer $m$ is characterized by the number of arcs of $\pi^{-1}(\pi(K))$ which converge to $p$, and is therefore independent of the choice of $V$ and $\gamma_{0}$. We define the order of ramification of $\pi$ at $p$ to be $o(p)=m-1$. Thus $o(p)>0$ if and only if $p$ is a ramified point of $\pi$, as follows from Proposition 2.

Corollary 2. Suppose $\pi: U \cup K \rightarrow M_{1}$ satisfies the hypotheses of Proposition 2. Then the set $B_{\text {ə }}$ of ramified boundary points is discrete.

Proof. According to Proposition 2, any point $p \in B_{\partial}$ has a neighborhood $D \cup K$ such that $D \cap \pi^{-1}(\pi(K))$ consists of a nonempty union of disjoint Jordan arcs tending to $p$ and having no other limit points on the boundary. But for $p^{\prime} \in K$ sufficiently close to $p, D \cup K$ is a neighborhood of $p^{\prime}$, so that $p^{\prime}$ is not the end point of any arc in $\pi^{-1}(\pi(K))$, and therefore $p^{\prime} \notin B_{\text {子. }}$.

We are now ready to prove our main result. It may be observed that the description of the behavior of $\pi$ given in Propositions 1 and 2 can be used to satisfy the hypotheses used by Elwin and Short in [2] to prove an Euler-characteristic formula similar to the one given below. For the sake of completeness, we shall give a proof relying only on elementary topological methods. 
THEOREM. Suppose $\bar{M}, \bar{M}_{1}$ are compact oriented surfaces-withboundary, $\pi: \bar{M} \rightarrow \bar{M}_{1}$ a continuous surjective mapping which is a branched immersion in $M$, and whose restriction to $\partial M$ is injective. Then (i) the set $B_{r} \subset \bar{M}$ of ramified points of $\pi$ is finite; (ii) the function $\operatorname{deg}(\pi)$ on $M_{1}$ has an upper bound $\mu$; and (iii) the Euler-characteristic formula

$$
\chi(\bar{M})+\sum_{p \in B_{r}} o(p)=\sum_{i=1}^{\mu} \chi\left(\bar{M}_{t}\right)
$$

holds, where for $p \in B=B_{r} \cap M, o(p)$ is defined in the introduction; for $p \in B_{\partial}=B_{r} \cap \partial M, o(p)$ is defined following Proposition 2; and for $i \geqq 1$, $M_{i}=\left\{p_{0} \in M_{1}: \operatorname{deg}(\pi)\left(p_{0}\right) \geqq i\right\}$.

Proof. Conclusion (ii) follows from Corollary 1. To obtain conclusion (i), we first use Proposition 1 to see that $B=B_{r} \cap M$ is finite. Now for any $p \in B_{\partial}=B_{r} \cap \partial M$, there is a neighborhood $U \cup K$ of $p$ in $\bar{M}$ disjoint from $B$ and which therefore satisfies the hypotheses of Corollary 2 , so that $p$ is an isolated point of $B_{\partial}$. Thus $B_{\partial}$ is discrete, and hence finite, since $\partial M$ is compact.

In order to verify formula $(*)$, we first modify $\pi$, if necessary, on a small neighborhood of each interior branch point, so that for $p, q \in B$, $\pi(p) \neq \pi(q)$; and for $p \in B, \pi(p) \notin \pi(\partial M)$. The modified mapping still satisfies all hypotheses and has the same order of branching at corresponding branch points. We list the boundary ramified points $B_{d}=\left\{p_{1}, \cdots, p_{n}\right\}$ and the interior branch points $B=\left\{q_{1}, \cdots, q_{\nu}\right\}$.

For each $p_{\imath} \in B_{\partial}$, taken in order, we may apply Proposition 2 in a neighborhood of $p_{\imath}$ disjoint from the finite set $B$, to see that there is a simply-connected neighborhood $D_{\imath} \cup K_{\imath}$ of $p_{\imath}$ in $\bar{M}$, with the following properties. (1) $D_{\imath}$ is bounded by the arc $K_{\imath}$ of $\partial M$ and a single Jordan arc in $M$. (2) The image $\pi\left(D_{1} \cup K_{t}\right)$ is an open disk in $M_{1}$, bounded by a Jordan curve which meets $\pi(\partial M)$ in exactly two points; $\pi\left(D_{\imath} \cup K_{\imath}\right)$ is separated into two simply-connected components by $\pi(\partial M)$, on one of which $\operatorname{deg}\left(\pi \mid D_{\imath}\right)$ has the constant value $o\left(p_{\imath}\right)+1$, and the constant value $o\left(p_{t}\right)$ on the other. (3) $D_{\imath} \cup K_{t}$ is small enough that $\overline{\pi\left(D_{\imath} \cup K_{\imath}\right)}$ is disjoint from $\overline{\pi\left(D_{1} \cup K_{\mathrm{I}}\right)}$ for $1 \leqq j<i$, from $\pi\left(p_{i}\right), i<j \leqq n$, and from $\pi(B)$.

We next take each interior branch point $q_{k} \in B$ in order. There is a simply-connected, open neighborhood $E_{k}$ of $q_{k}$ in $M$, bounded by a single Jordan curve in $M$, with the following properties. (1) $\pi\left(E_{k}\right)$ is a disk in $M_{1}$, bounded by a Jordan curve in $M_{1}$. (2) deg $\left(\pi \mid E_{k}\right)$ has the constant value $o\left(q_{k}\right)+1$ on $\pi\left(E_{k}\right)$. (3) $\overline{\pi\left(E_{k}\right)}$ is disjoint from $\overline{\pi\left(D_{i} \cup K_{l}\right)}, 1 \leqq i \leqq$ $n$, from $\pi\left(E_{\jmath}\right), 1 \leqq j<k$, and from $\pi\left(q_{j}\right), k<j \leqq \nu$. Namely, there are neighborhoods $V$ of $q_{k}$ in $M$ and $V_{0}$ of $\pi\left(q_{k}\right)$ in $M_{1}$, and homeomorphisms $g: V \rightarrow \Delta, g_{0}: V_{0} \rightarrow \Delta$ onto the unit disk, such that $g_{0}(\pi(p))=$ $(g(p))^{m}$ for all $p \in V$, where $m=o\left(q_{k}\right)+1$. Therefore, we may choose 
$E_{k}=\{p \in V:|g(p)|<\epsilon\}$ for a sufficiently small $\epsilon>0$. Observe that $\overline{\pi\left(D_{1} \cup K_{1}\right)}, \cdots, \pi\left(D_{n} \cup K_{n}\right), \overline{\pi\left(E_{1}\right)}, \cdots, \overline{\pi\left(E_{\nu}\right)}$ are disjoint closed disks in $M_{1}$.

We now define a topological surface-with-boundary $\bar{\Sigma}=$ $\bar{M} \backslash \bigcup_{j=1}^{n} D_{J} \backslash \bigcup_{k=1}^{v} E_{k}$. Then $\pi$ is a local homeomorphism on $\bar{\Sigma}$, although not an open mapping in general. For convenience, we let $M^{\prime}$ denote the disjoint union $M_{1}+\cdots+M_{\mu}$, where $M_{i}=\left\{p \in M_{1}: \operatorname{deg}(\pi) \geqq i\right\}$. Then $\chi\left(\bar{M}^{\prime}\right)=\Sigma_{i=1}^{\mu} \chi\left(\bar{M}_{i}\right)$. Similarly, $\Sigma^{\prime}=\Sigma_{1}+\cdots+\Sigma_{\mu}$, where $\Sigma_{i}=\left\{p \in M_{1}\right.$ : $\operatorname{deg}(\pi \mid \Sigma) \geqq i\} . M^{\prime}$ and $\Sigma^{\prime}$ may be thought of as the leaves of the branched coverings $\pi$ and $\pi \mid \Sigma$, respectively. Thus a regular value $p_{0} \in M_{1}$ of $\pi$ appears once in $M^{\prime}$ for each point of the fiber $\pi^{-1}\left(p_{0}\right) \cap M$.

Observe that $\chi(\bar{\Sigma})=\chi\left(\bar{\Sigma}^{\prime}\right)$. In fact, we may triangulate $\bar{\Sigma}_{1}$ in such a way that $\bar{\Sigma}_{2}, \cdots, \bar{\Sigma}_{\mu}$ are subcomplexes, and give $\bar{\Sigma}$ the triangulation induced by the local homeomorphism $\pi$. Then a simplex of $\bar{\Sigma}_{1}$ occurs in $\bar{\Sigma}^{\prime}$ exactly as many times as there are simplices in $\bar{\Sigma}$ mapped onto it. Now $\bar{\Sigma}^{\prime}$ is obtained from $\bar{M}^{\prime}$ by removing certain interior disks and boundary half-disks: for each $p_{\jmath} \in B_{\partial}, o\left(p_{l}\right)$ interior disks and one boundary half-disk is removed, while for each $q_{k} \in B, o\left(q_{k}\right)+1$ interior disks are removed. This gives a total of $0+\nu$ interior disks and $n$ boundary half-disks, where $0=\sum_{p \in B_{r}} o(p)$ is the total order of ramification of $\pi$. Therefore, one may compute $\chi\left(\bar{\Sigma}^{\prime}\right)=\chi\left(\bar{M}^{\prime}\right)-(0+\nu)$. In fact, $\bar{\Sigma}^{\prime}$ and the closure $T$ of its complement in $\bar{M}^{\prime}$ are simplicial subcomplexes, so that

$$
\chi\left(\bar{M}^{\prime}\right)+\chi\left(\bar{\Sigma}^{\prime} \cap T\right)=\chi\left(\bar{\Sigma}^{\prime}\right)+\chi(T)
$$

(cf. [6], pp. 189-90). But $\chi(T)=0+n+\nu$ and $\chi\left(\bar{\Sigma}^{\prime} \cap T\right)=n$. Similarly, one may compute $\chi(\bar{\Sigma})=\chi(\bar{M})-\nu$. Therefore

$$
\chi(\bar{M})+0=\chi(\bar{\Sigma})+0+\nu=\chi\left(\bar{M}^{\prime}\right)=\sum_{i=1}^{\mu} \chi\left(\bar{M}_{i}\right) .
$$

We have not treated the question of a topological characterization of mappings which are branched immersions up to the boundary and which map the boundary injectively. A mapping $\pi: M \rightarrow M_{1}$ between surfaces may be called a branched immersion up to the boundary if $\pi \mid M_{1}$ is a brançhed immersion and moreover, for every boundary point $p$, there is an integer $m=o(p)+1$, a neighborhood $V$ of $p$ in $\vec{M}$, a neighborhood $V_{0}$ of $\pi(p)$ in $M_{1}$ and homeomorphisms $g: V \rightarrow \Delta^{+} \cup I, g_{0}: V_{0} \rightarrow \Delta$, such that for all $q \in V, g_{0}(\pi(q))=(g(q))^{2 m-1}$. Here $\Delta, \Delta^{+}$, and $I$ are as in Lemma 1. It seems likely that a mapping satisfying the hypothesis of the theorem may be shown to be a branched immersion up to the boundary, using the result of Proposition 2. We shall be satisfied here with the 
following description of the set $\pi^{-1}(\pi(\partial M))$. The proof follows immediately from Proposition 2.

Corollary 3. Suppose $\pi: \bar{M} \rightarrow \bar{M}_{1}$ satisfies the hypotheses of the theorem. Then $\pi^{-1}(\pi(\partial M))$ consists of $\partial M$ along with a finite union of Jordan curves and arcs in $M$, plus the finite set $B=B_{r} \cap M$. Each such Jordan arc tends at each end to a point of $B_{r}$. Each ramified point $p \in B_{r}$ is the endpoint of $2 o(p)+2$ arcs of $\pi^{-1}(\pi(\partial M))$, including, for $p \in B_{\partial}$, the two adjoining arcs of $\partial M$.

\section{REFERENCES}

1. L. Ahlfors, Zur Theorie der Überlagerungsflächen, Acta Math., 65 (1935), 157-194.

2. J. Elwin and D. Short, Branched immersions onto compact orientable surfaces, Pacific J. Math., 54 (1974), 113-122.

3. R. Gulliver, Branched immersions of surfaces and reduction of topological type, I: Math. Z., 145 (1975), 267-288. II: (preprint).

4. R. Gulliver, R. Osserman and H. Royden, A theory of branched ımmersions of surfaces, Amer. J. Math., 95 (1973), 750-812.

5. J. Milnor, Topology from the Differentiable Viewpoint, University Press of Virginia, Charlottesville, 1965.

6. E. Spanier, Algebraic Topology, McGraw-Hill, New York, 1966.

7. S. Stoilow, Leçons sur les principes topologiques de la théorie des fonctions analytiques, Gauthier-Villars, Paris, 1938.

Received May 20, 1975.

UniVersity of MinNesota 




\section{Pacific Journal of Mathematics}

Vol. 64, No. 1

May, 1976

Walter Allegretto, Nonoscillation theory of elliptic equations of order $2 n \ldots \ldots \quad 1$

Bruce Allem Anderson, Sequencings and starters.................. 17

Friedrich-Wilhelm Bauer, A shape theory with singular homology .......... 25

John Kelly Beem, Characterizing Finsler spaces which are

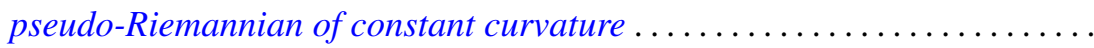

Dennis K. Burke and Ernest A. Michael, On certain point-countable

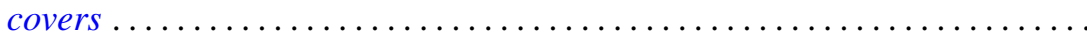

Robert Chen, A generalization of a theorem of Chacon ............... 93

Francis H. Clarke, On the inverse function theorem ................ 97

James Bryan Collier, The dual of a space with the Radon-Nikodým

property ....................................... 103

John E. Cruthirds, Infinite Galois theory for commutative rings ............ 107

Artatrana Dash, Joint essential spectra......................... 119

Robert M. DeVos, Subsequences and rearrangements of sequences in FK

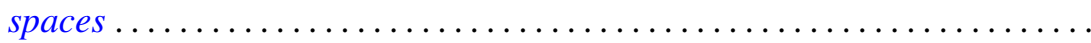

Geoffrey Fox and Pedro Morales, Non-Hausdorff multifunction generalization

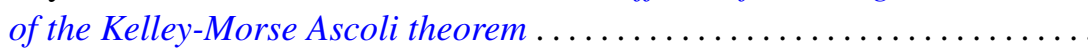

Richard Joseph Fleming, Jerome A. Goldstein and James E. Jamison, One

parameter groups of isometries on certain Banach spaces.............

Robert David Gulliver, II, Finiteness of the ramified set for branched

immersions of surfaces

Kenneth Hardy and István Juhász, Normality and the weak cb property ...... 167

C. A. Hayes, Derivation of the integrals of $L^{(q)}$-functions.

Frederic Timothy Howard, Roots of the Euler polynomials .

Robert Edward Jamison, II, Richard O'Brien and Peter Drummond Taylor, On

embedding a compact convex set into a locally convex topological vector space ....................................

Andrew Lelek, An example of a simple triod with surjective span smaller than span ...

Janet E. Mills, Certain congruences on orthodox semigroups

Donald J. Newman and A. R. Reddy, Rational approximation of $e^{-x}$ on the positive real axis.

John Robert Quine, Jr., Homotopies and intersection sequences ...

Nambury Sitarama Raju, Periodic Jacobi-Perron algorithms and fundamental units ....

Herbert Silverman, Convexity theorems for subclasses of univalent functions. . .

Charles Frederick Wells, Centralizers of transitive semigroup actions and endomorphisms of trees.........................

Volker Wrobel, Spectral approximation theorems in locally convex spaces ..................... 